\title{
Antimatter and antistars in the universe and in the Galaxy
}

\author{
S.I. Blinnikov, ${ }^{1,2,3, * \text { A.D. Dolgov, }}{ }^{1,2,4, \dagger}$ and K. A. Postnov ${ }^{5}$, 田 \\ ${ }^{1}$ Novosibirsk State University, Novosibirsk, 630090, Russia \\ ${ }^{2}$ ITEP, Bol. Cheremushkinsaya ul., 25, 113259 Moscow, Russia \\ ${ }^{3}$ Kavli IPMU (WPI), Tokyo University, Kashiwa, Chiba, Japan \\ ${ }^{4}$ Dipartimento di Fisica e Scienze della Terra, Università degli Studi di Ferrara \\ Polo Scientifico e Tecnologico - Edificio C, Via Saragat 1, 44122 Ferrara, Italy \\ ${ }^{5}$ Moscow M.V. Lomonosov State University, Sternberg Astronomical Institute
}

We consider consequences of hypothetical existence of baryo-dense stars created in the very early universe within an extension of Affleck-Dine scenario of baryogenesis. New constraints on the possible number of compact antimatter objects are derived. The contemporary observational data do not exclude significant amount of antimatter in the Galaxy (and in other galaxies) in the form of the baryo-dense low-massive stars.

\footnotetext{
* Sergei.Blinnikov@itep.ru

† dolgov@fe.infn.it

$\ddagger$ pk@sai.msu.ru
} 


\section{INTRODUCTION}

Despite almost identical properties of particles and antiparticles, all matter observed in our neighborhood consists only of particles, i.e. of protons, neutrons and electrons. A small fraction of antiprotons in cosmic rays, about $10^{-4}$ with respect to protons, most probably can be explained by their secondary origin. Predominance of matter over antimatter was beautifully explained by Sakharov [1] as dynamically generated in the early universe due to breaking of $\mathrm{C}$ and $\mathrm{CP}$ invariance, non-conservation of the baryonic number, and violation of thermal equilibrium.

On the other hand, there are plenty of theoretical possibilities leading to abundant creation of antimatter in the universe. For example, if CP-invariance is broken spontaneously [2], the universe would be equally populated by matter and antimatter. However, in this case the nearest antimatter domain should be practically at the cosmological horizon, at a few gigaparsec distance [3]. Still less pessimistic scenarios are possible and independently of the theory, a search for real (not secondary produced) cosmic antimatter should be done and is being performed by several detectors, including BESS [4] PAMELA [5], and AMS [6] More detectors are in project.

An unambiguous proof of existence of the primordial antimatter would be an observation of sufficiently heavy antinuclei, starting from ${ }^{4} \mathrm{He}$. According to theoretical estimates [7] antideuterium could be created in the energetic cosmic ray reactions of $\bar{p} p$ or $\bar{p}$ He collisions with a flux of $\sim 10^{-7} \mathrm{~m}^{2} / \mathrm{s}^{-1} / \mathrm{sr} /(\mathrm{GeV} / \mathrm{n})$, i.e. five orders of magnitude lower than the observed flux of antiprotons. The fluxes of the secondary-produced $\overline{{ }^{3} \mathrm{He}}$ and $\overline{{ }^{4} \mathrm{He}}$ are predicted to be much smaller, respectively four and eight orders of magnitude below that of antideuterium. Experimental search of antinuclei production is performed at LHC by ALICE Collaboration. The results can be found in [8] and are reported at a seminar by A.P. Kalweit [9]. Though the production rate looks significant, with the suppression factor about $1 / 300$ per each extra (anti)nucleon added to a nuclei, such events are quite rare in cosmology and their contribution to the total cosmological production is very small.

As for the astronomical observations, at the present time there are only upper bounds on the flux of cosmic antihelium-4 [4]: $\overline{\mathrm{He}} / \mathrm{He}<3 \times 10^{-7}$. In the nearest future this bound is expected to be improved down to $\overline{\mathrm{He}} / \mathrm{He}<3 \times 10^{-8}\left[\underline{5}\right.$ ] and $\overline{\mathrm{He}} / \mathrm{He}<10^{-9}[\underline{6}$.

There is another direction for search of cosmic antimatter through analysis of cosmic electromagnetic radiation, in particular of $\sim 100 \mathrm{MeV}$ photons from $\bar{p} p$-annihilation and of the $0.511 \mathrm{MeV}$-line from $e^{+} e^{-}$annihilation at low energies. According to these data, the bounds on the fraction of antimatter in several galaxies, in particular on the amount of antistars, is generally below $10^{-6}$ of the total amount of matter there. The absence of excessive gamma radiation allows one to conclude that the nearest anti-galaxy could not be closer than $\sim 10 \mathrm{Mpc}$ [10]. The mass fraction of antimatter in two observed colliding galaxies in the Bullet cluster cannot be larger than 10 ${ }^{-6}[11]$. As for our Galaxy, it is shown in ref. [12], that the amount of antistars is bounded by $N_{*} / N_{*}<4 \cdot 10^{-5}$ within 150 pc from the Sun.

The quoted limits are valid if the antimatter objects are of the same kind as those made of the ordinary matter. However, they may be essentially different, as argued in refs [13, 14], where an efficient mechanism of the cosmological antimatter creation was suggested and studied. According to this model, which is discussed below to make the paper self-contained, the antistars could be formed in the very early universe as compact objects, which may be predominantly dead now. They are not concentrated in galaxies but distributed in a larger volume, in the halo, and have larger velocities than the normal stars. In this sense, they are similar to cold dark matter particles. In such a case the restrictive limits derived for the "normal" antistars are not applicable and such new type antimatter objects may abundantly populate the Galaxy. Phenomenology of such antimatter objects is discussed in ref. [15]. As antistars may be abundant in the Galaxy, it is of interest to consider the possibility of their registration by terrestrial detectors which at the present time are sensitive only to relatively close objects. It was shown in ref. [16] that the width of different atomic lines are different for atoms and antiatoms, but the effect is too weak to be observed in the foreseeable future. More promising for a registration of antistars in the Galaxy are measurements of polarization of some lines of the stellar electromagnetic radiation, especially in the nuclear transitions or of neutrino versus antineutrino fluxes [17]. There is no chance to see anti-stars in distant galaxies except for a lucky case of a star-antistar collision which would be energetic enough to be observed on earth.

In ref. [18] we applied the mechanism of the early star formation, which is used below for creation of antimatter, for an explanation of existence of quasars/supermassive black holes, gamma-ray bursters and supernovae at high redshifts, as well as of stars in the Galaxy, which look older than the universe.

Some other models of the antimatter creation can be found in reviews [19, 20]. The current observational bounds are also extensively reviewed in refs. [12, 21]

The standard scenarios of baryogenesis deal with only one number, namely with the ratio of the baryonic number density to the density of photons in the microwave background radiation. Their usual outcome is a homogeneous baryon asymmetry all over the universe. So there is no way to discriminate between different models having only one number in possession. In this connection the models of baryogenesis which lead to noticeable isocurvature perturbations and especially domains with negative asymmetry, i.e. antimatter domains, are of great interest and an astronomical search of cosmic antimatter and a study of theoretical scenarios of antimatter creation become of primary importance in the attempts to understand generation of the cosmological matter-antimatter asymmetry. 
The paper is organized as follows. In sec. III to make the presentation self-contained, we discuss the main features of the model leading to an efficient production of antimatter objects (antistars, dense clouds of antimatter) which may "live" in the Galaxy in a significant amount. Section III is devoted to estimates of possible abundance of antimatter in diffusive interstellar state. We derive new constraints on the possible number of compact antimatter objects in sec. [V] Discussion and conclusions are presented in sec. D.

\section{A MECHANISM OF STAR AND ANTISTAR FORMATION IN THE EARLY UNIVERSE}

All scenarios of baryogenesis, but one, normally predict rather low values of the baryon asymmetry, so theoretical efforts are aimed at increasing the prediction up to the largest possible number to obtain the observed magnitude of the baryon asymmetry

$$
\beta_{\text {obs }}=n_{B} / n_{\gamma} \approx 6 \cdot 10^{-10}
$$

The only exception is the model suggested by Affleck and Dine (AD) [22] where the cosmological baryon asymmetry is normally much larger than the observed value, and efforts should be done in the opposite direction to diminish it down to (2.1).

The AD-scenario of baryogenesis is based on high-energy supersymmetric (SUSY) model which naturally possesses flat directions (valleys) in the potential of scalar superpartner of baryons. Such a scalar field has nonzero baryonic number and could acquire a large vacuum expectation value during inflation, if its mass is smaller than the Hubble parameter at that period. After inflation was over, the baryonic number density accumulated in the rotational motion of the Affleck-Dine field, $\chi$, i.e. in its time dependent phase, is transformed into baryonic number of quarks through baryo-conserved decays of $\chi$.

The essential features of the potential of $\chi$ can be described by the following toy model expression:

$$
U(\chi)=\lambda\left(2|\chi|^{4}+\chi^{4}+\chi *^{4}\right)+\left(m_{1}^{2} \chi^{2}+\text { h.c. }\right)+m_{2}^{2}|\chi|^{2},
$$

where the mass parameter $m_{1}$ may be complex, leading to $\mathrm{C}$ and $\mathrm{CP}$ violation if $\lambda \neq 0$; $m_{2}$ may be taken equal to $2\left|m_{1}\right|$ to avoid non-zero vacuum expectation value of $\chi$. A positive $m_{2}^{2}$ may appear as a result of some spontaneous symmetry breaking at a later stage of the cosmological evolution. It is analogous to the well known Higgs phenomenon, The first two terms in the potential have flat directions, along which the potential does not grow, but for complex $m_{1}$ the the flat directions of the quadratic term do not coincide with quartic ones. Because of one-loop radiative corrections the quartic terms can acquire logarithmic factors of the type $\sim \chi^{4} \ln \left(|\chi|^{2} / \sigma^{2}\right)[23]$.

During inflation, when the Hubble parameter was much larger than $\left|m_{1}\right|$ and $\left|m_{2}\right|$, the amplitude of field $\chi$ can reach high magnitude and when inflation was over and the Hubble parameter became smaller than the slope of the potential, $\chi$ started evolving down to the origin and on the way it could acquire a large "angular momentum" in the complex $[\operatorname{Re} \chi, \operatorname{Im} \chi]$-plane due to misalignment of the quartic and quadratic flat directions. The angular momentum is essentially the baryonic number accumulated by $\chi$ :

$$
n_{B}(\chi)=i\left[\chi^{*}\left(\partial_{t} \chi-\left(\partial_{t} \chi^{*}\right) \chi\right]\right.
$$

If there is no phase difference between $m$ and $\lambda$ and a quadratic flat direction coincides with a quartic one, then there would be no misalignment but nevertheless a non-zero angular momentum could be induced due to quantum fluctuations of $\chi$ in the direction orthogonal to the valley. In this case baryonic density would be also created but with different signs in different space domains because of the chaotic behavior of quantum fluctuations. So on average the baryon asymmetry would be zero.

This simple AD-scenario was slightly modified in ref. [13] by an addition of the general renormalizable coupling of $\chi$ to inflaton field $\Phi$ :

$$
\delta U(\chi, \Phi)=g|\chi|^{2}\left(\Phi-\Phi_{1}\right)^{2}
$$

where $\Phi_{1}$ is supposed to be the value which $\Phi$ passed during inflation not too close to its end. It allows the size of the bubbles with high baron asymmetry to become astronomically large. This is the only tuning parameter of the model. An essential effect created by the addition of $\delta U$ to the potential of $\chi$ is that the window to the flat directions is open only for a short time when $\Phi$ is close to $\Phi_{1}$, so the probability to reach a high value for $\chi$ would be small. Correspondingly $\chi$ would be large only in a small fraction of space. In this case cosmologically small but possibly astronomically large bubbles with high $\beta$ could be created, occupying a small part of the universe volume, while the rest of the universe would have normal $\beta \approx 6 \cdot 10^{-10}$, created by a small $\chi$ which occupied the bulk of space. Nevertheless the fraction of baryonic and antibaryonic matter in this compact objects may exceed that of the observed baryons. In the simplest version of the model the amount of baryons and antibaryons in high $\beta$ regions would be equal, but in more general case their ratio is model-dependent and may be arbitrary. 
The bubbles with high values of $\chi$ after B-conserving decay of $\chi$ into fermions would form domains with a large baryonic number density in the form of the usual quark/baryon matter. The rest of the universe would have normal small baryon asymmetry. Initially the density contrast between the regions with low and high values of $\chi$ was zero or very small (isocurvature perturbations). After formation of the domains with large $\chi$ the equation of state inside and outside the bubbles became different because the matter inside the bubbles was more non-relativistic than the matter outside. This would create some initial density contrast inside and outside high-B bubbles. For this and the following (next paragraph) conclusion it is essential that the baryon diffusion length was very short.

The second period of generation of the density contrast inside and outside the bubbles, $\delta \varrho$, took place after the QCD phase transition at temperatures somewhat above $100 \mathrm{MeV}$, when quarks formed non-relativistic protons. At this stage a whole family of compact stellar-like objects with baryon number density much higher than the background baryon density had been formed. Depending upon the relation between their mass and the corresponding Jeans mass they could be very early stars, progenitors of Supernovae, or primordial black holes. The mass distribution of these objects has the log-normal form:

$$
\frac{d N}{d M}=C_{M} \exp \left[-\gamma \ln ^{2}\left(M / M_{0}\right)\right]
$$

where $C_{M}, \gamma$, and $M_{0}$ are constant model-dependent parameters. The form of the spectrum is practically modelindependent since it is determined by the exponential expansion. We call these regions as baryo-dense (BD) ones and the stars formed in such regions can be called BD-stars and even BD-black holes.

In particular, primordial black holes with masses from a few solar masses up to $10^{6-7} M_{\odot}$, or even more (on the tail of the distribution), can be created. Such superheavy black holes could be seeds of galaxy formation. It is easy to choose the parameters of the model that there would be one superheavy black hole (BH) for any existing large galaxy. This scenario offers a new mechanism of the early superheavy BH (quasar) formation and ultracompact dwarf galaxies where the $\mathrm{BH}$ may have more than $10 \%$ of the mass of the whole galaxy [24]. At the present time there is no satisfactory explanation for the early formation of superheavy $\mathrm{BH}$ and ultracompact dwarf galaxies with large BHs in the framework of the conventional theories. Moreover, in the model considered here a natural explanation is found for existence of high red-shift gamma-ray bursters, of early supernovae, of the metal- enriched chemistry in the vicinity of those early-formed objects, and of the stars which are formally older that the universe. These problems are discussed in an earlier publication by two of us [18]. In this work we dwell on phenomenology, observational manifestations, and bounds on antistars, which came to us from the early universe and might populate the galaxy.

Of course big bang nucleosynthesis (BBN) in the regions with high $\beta$ would be significantly different from the standard one with low $\beta$; much more heavy elements would be produced there. The calculations of the element abundances created at BBN with high $\beta$ have been done in ref. [25], but unfortunately only with $\beta \ll 1$, though with $\beta \gg \beta_{\text {obs }}$. It would be very interesting to extend such calculations up to $\beta \geq 1$. One immediate effect is that the hydrogen to helium ratio would significantly drop down because for large $\beta$ the neutron-proton freezing took place at higher temperatures when $n / p$ ratio was close to one. So the stars formed after BBN in high-B bubbles would mostly consist of ${ }^{4} \mathrm{He}$ plus some metals which are normally absent in first stars. Since in the simplest version of the scenario the baryo-dense objects consist of matter and antimatter in roughly equal number, an anomaly in elemental abundances somewhere in the Galaxy could be an indicator of antimatter there with $50 \%$ probability. On the other hand, the observed abundances of light elements created at BBN would not be significantly different from the predictions of the standard theory with low $\beta$, because the low- $\beta$ volume is much larger than that with high $\beta$ and the clouds with anomalous abundances are quite rare.

As is well known, the spectrum of angular fluctuations of CMB measures $\beta$ quite close to (2.1). However, it does not exclude BD-objects considered here because the anomalies in the baryonic number density occur at very low scales much shorter than tens megaparsecs to which the CMB measurements are sensitive.

In what follows we put aside theory and consider all possible observational manifestations of antimatter in the Galaxy not restricting ourselves to any particular forms of antimatter objects, using the principle "everything which is not forbidden is allowed".

\section{CONSTRAINTS FROM DIFFUSIVE ANTI-MATTER}

Let us start with the simplest constraint on diffusive anti-matter in the universe from the production of highenergy photons (see also [10, 12]).

The observed isotropic EGRET background [26] in the $\sim 100 \mathrm{MeV}$ energy range can be approximately represented as

$$
I(100 \mathrm{MeV}) \approx 1000\left[\mathrm{eV} / \mathrm{cm}^{2} / \mathrm{s} / \mathrm{ster}\right]
$$


This corresponds to the energy density of $100 \mathrm{MeV}$ photons:

$$
\epsilon_{\text {obs }}(100 \mathrm{MeV})=\frac{4 \pi}{c} I(100 \mathrm{MeV}) \approx 4 \times 10^{-7}\left[\mathrm{eV} / \mathrm{cm}^{3}\right]
$$

Baryons in stars amount to $\Omega_{b}^{*} \simeq 10^{-3}$ [27] (in units of the critical density in the Universe), which corresponds to the energy density

$$
\epsilon^{*}=\Omega_{b}^{*} \varrho_{c r} c^{2} \sim 5.2\left[\mathrm{eV} / \mathrm{cm}^{3}\right]
$$

For the sake of a simple estimate we assume that that most of the stars, including the BD ones, are similar to the Sun, so they lose about $50 \%$ of their mass in due course of evolution. Adopting an equal amount of BD antimatter stars, $\Omega_{\widetilde{b}}=\Omega_{b}^{*}$, and allowing for the maximum annihilation of matter and antimatter during the Hubble time, we get a rough upper limit on the spatial mixing of diffuse matter/antimatter, $f<3 \times 10^{-8}$, in order to respect the observed gamma-ray background.

Note that unlike the usual stars, the BD stars can initially be compact and consist of anti-helium, so their evolutionary mass loss can be much smaller than for the ordinary stars (see [28, 29] and the discussion below). Moreover, this mass loss took place at high redshift, probably at $z>10$, and the flux of the photons from matter and antimatter annihilation would be strongly reduced and shifted to smaller energies. Therefore, it is necessary to consider another limiting case where the primordial antimatter survived in the form of compact objects.

\section{CONSTRAINTS FROM COMPACT ANTI-MATTER OBJECTS}

\section{A. Annihilation at accretion}

A compact BD-star with mass $M$ passing through diffusive interstellar or intergalactic medium with number density $n_{0}$ will accrete baryonic matter. According to the well-known Bondi-Hoyle-Littleton formula, the accretion rate is

$$
\dot{M} \simeq\left(\frac{2 G M}{v^{2}}\right)^{2} m_{p} n_{0} v \approx 10^{11}[\mathrm{~g} / \mathrm{s}]\left(\frac{M}{M_{\odot}}\right)^{2}\left(\frac{n_{0}}{1 \mathrm{~cm}^{-3}}\right)\left(\frac{v}{10 \mathrm{~km} / \mathrm{s}}\right)^{-3}
$$

This accretion rate exactly corresponds to the widely used formula (23) from ref. [10] for photonic luminosity due to annihilation of accreting matter.

As mentioned in Section I, compact BD stars can be treated as cold dark matter particles. That is, they should have virial velocities in galaxy halos about $v_{\mathrm{BD}} \sim 500 \mathrm{~km} \mathrm{~s}^{-1}$. Unfortunately the galactic escape velocity and hence the virial velocity are not well known [30, 31]. For a recent review see ref. 32. The value $v_{\mathrm{esc}}=650 \mathrm{~km} \mathrm{~s}$ (90\% upper confidence limit) from ref, 33] is usually taken. Ref. 34] finds the updated escape speed in the range $498 \mathrm{~km} \mathrm{~s}^{-1}<v_{\text {esc }}<608 \mathrm{~km} \mathrm{~s}^{-1}$ at $90 \%$ confidence, with a median likelihood of $v_{\text {esc }}=544 \mathrm{~km} \mathrm{~s}^{-1}$.

Therefore, the gas accretion rate onto a compact BD-star is dramatically decreased, $\dot{M}_{\mathrm{BD}} \sim 10^{6} \mathrm{~g} / \mathrm{s}$ if we take safe realistic values $v_{\mathrm{BD}} \sim 300-500 \mathrm{~km} \mathrm{~s}^{-1}$. This implies that BD-stars comply with the constraints considered in ref [12].

At this low accretion luminosity $\left(\sim 10^{27}-10^{28} \mathrm{erg} / \mathrm{s}\right.$, which may be lower than their intrinsic luminosity), BD-stars are very difficult to discover. They may appear as rapidly moving cold objects, for example, in the forthcoming GAIA or WSRT observations.

\section{B. Binary BD stars}

Consider now a possible case of a binary BD-star consisting of antimatter. In the process of evolution, the stars in the system could coalesce due to gravitational wave emission to produce an explosive event like ordinary type Ia supernova. This explosion would inject around 1-2 solar masses of antimatter into the interstellar medium and produce a supernova remnant (SNR). In addition to ordinary thermal shock-wave emission (mostly in keV range), in this case one should expect a high flux of hard photons from $e^{+} e^{-}$and proton-antiproton annihilation in the interstellar medium.

In reality, the mean free-path of an (anti)baryon is determined by the magnetic field which is inevitably generated behind the shock front $[35]$ 39]. For an estimate, assume $B \equiv 10^{-5} \mathrm{G} \times B_{-5}$. The Larmor radius for a proton is

$$
r_{L}=(v / c) m_{p} c^{2} / e B=10^{10}[\mathrm{~cm}] B_{-5}^{-1} v_{9},
$$

where the shock velocity, $v_{9}$, is normalized to $10000 \mathrm{~km} / \mathrm{s}$. 
Let us consider a young anti-SNR with radius $R=10^{18} \mathrm{~cm} R_{18}$. The mass inside the layer of active annihilation is equal to:

$$
\Delta M=4 \pi R^{2} r_{L} m_{p}\left(4 n_{0}\right)
$$

where $n_{0} \sim 1 \mathrm{~cm}^{-3}$ is the ambient ISM number density. The annihilation time is $t_{\text {ann }}=1 /(n \sigma v)$, where the annihilation cross-section is inversely proportional to the center of mass velocity of the colliding proton-antiproton pair and is given by $\sigma v \sim 10^{-15} \mathrm{~cm}^{3} / \mathrm{s}$. So the annihilation time is:

$$
t_{\mathrm{ann}} \approx 10^{15}[\mathrm{~s}]\left(n / \mathrm{cm}^{3}\right)^{-1}
$$

In the case of very slow relative velocity of protons and antiprotons, e.g. $v \sim 10^{-3}$, the cross-section is enhanced approximately by an order of magnitude due to Coulomb attraction between $p$ and $\bar{p}$ (Sommerfeld enhancement). Thus, the annihilation luminosity is

$$
L_{\mathrm{ann}}=\frac{\Delta M c^{2}}{t_{\mathrm{ann}}} \approx 5 \cdot 10^{29}[\mathrm{erg} / \mathrm{s}] R_{18}^{2} B_{-5}^{-1}\left(n / \mathrm{cm}^{3}\right)^{2} .
$$

The absolute upper limit on the annihilation luminosity is $\sim M_{\odot} c^{2} / t_{\mathrm{ann}} \approx 10^{39} \mathrm{erg} / \mathrm{s}$, but, of course, the estimate (4.5) is much closer to reality.

There can be also binaries formed of a star and an antistar by the gravitational capture through a threebody interaction with another star. The probability of such binary formation is possibly much smaller than the probability of formation of a binary consisting of two antistars which could be created from the same cloud with high baryon density. Still it is nonzero, and merging of antistar and star would lead to a spectacular explosion (hypernova?).

\section{Microlensing}

Compact BD-stars can be also found by the effect of gravitational microlensing which may be caused by both visible and invisible stars. These objects are called now MACHOs for "Massive Astrophysical Compact Halo Object". This phenomenon was first discussed in relation with DM candidates made of the so-called Mirror Matter by Berezhiani, Dolgov, and Mohapatra [40] and Blinnikov [41]).

\section{MACHO, EROS, AGAPE, MEGA, OGLE - contradicting results}

MACHO group [42] has revealed 13 - 17 microlensing events in the Large Magellanic Cloud (LMC), significantly higher number than that expected from the known stars but not enough to explain all dark matter (DM) in the halo. The fractional contribution of the objects which produced the lensing into the dark matter density is usually denoted as $f$. Such objects have also got the name Machos. MACHO group concluded that compact objects in the mass range $0.15 M_{\odot}<M<0.9 M_{\odot}$ have a fraction $f$ in galactic halo in the range $0.08<f<0.50(95 \% \mathrm{CL})$. So Bennett [43] has concluded (based on the results of MACHO group) that Machos have been really found.

EROS (Expérience pour la Recherche d'Objets Sombres) collaboration has placed only an upper limit on the halo fraction, $f<0.2(95 \% \mathrm{CL})$ for the objects in the specified above MACHO mass range, while EROS-2 [44] gives $f<0.1$ for $10^{-6} M_{\odot}<M<1 M_{\odot}$.

AGAPE collaboration [45], working on microlensing in M31 (Andromeda) galaxy, finds the halo Macho fraction in the range $0.2<f<0.9$. while MEGA group marginally conflicts with them with an upper limit $f<0.3$ [46].

Detailed analysis of the controversial situation with the results of different groups is given in ref. [47]. Newer results [48] for EROS-2 and OGLE (Optical Gravitational Lensing Experiment) in the direction of the Small Magellanic Cloud are: $f<0.1$ obtained at $95 \%$ confidence level for Machos with the mass $10^{-2} M_{\odot}$ and $f<0.2$ for Machos with the mass $0.5 M_{\odot}$.

Recent data on the other aspects of the microlensing are discussed in ref. [49].

It would be exciting if all DM were constituted by BD-stars (and BD black holes) with masses in still allowed intervals, but more detailed analysis of this possibility has to be done.

\section{Destruction of wide pairs of visible stars}

Paper [50], which appeared in the series "End of MACHO era (1974-2004)", asserts that wide pairs of visible stars must be destroyed by invisible Machos flying near them. The same effect may take place in the case of BD-stars (which are visible, but weak). In addition to the criticism of the paper [50], put forward in [51], one can 
point out that it is necessary to consider not only the process of destruction, but also a reverse process of creating pairs of visible stars from single individual stars not bound previously by the mutual gravity.

The probability of microlensing [52, 53] is naturally measured by the so-called optical depth $\tau$. Evans and Belokurov [51] confirmed lower number of compact objects in the direction to LMC, than obtained in the MACHO group, i.e. they got $\tau<0.36 \times 10^{-7}$ in agreement with EROS results [44]. Later, however, a paper of the same Cambridge group [54] was published where, on the basis of studies of binary stars, arguments in favor of real existence of Machos and against the pessimistic conclusions of ref. [50] were presented.

\section{Reionization and CMB}

Energy influx from matter-antimatter annihilation at high redshift, $z>10$ could be a source of the cosmological reionization, for which not enough energy is found in the standard model. Another way around, we can obtain a bound on the amount of antimatter annihilating at high redshifts, if no energy injection is observed. A simple constraint can be derived as follows. According to eq. (4.4) the annihilation time in the early universe is

$$
t_{\text {ann }}(z) \sim 0.5 \cdot 10^{23}[\mathrm{~s}](1+\mathrm{z})^{-3}
$$

Here we took for the baryon number density the value $n=\Omega_{b} \varrho_{c} / m_{p} \approx 2 \cdot 10^{-7}(z+1)^{3}$. An account of the Sommerfeld enhancement, which is effective at low velocities, $v<10^{-3}$, could enlarge the cross-section by an order of magnitude and correspondingly diminish $t_{\text {ann }}$ by the same factor.

At redshift $z=10$ the annihilation time is about $4 \cdot 10^{19}$ seconds, which is much longer than the universe age at this redshift, $t_{U}(z=10) \approx 10^{16} \mathrm{~s}$. So one may expect that about $10^{-3}-10^{-4}$ of all diffuse antimatter could annihilate, producing mostly $100 \mathrm{MeV}$ photons, energetic electrons and positrons. In principle there would be more than enough energy to reionize the universe but more detailed calculations of the rate of energy degradation down to atomic resonance is necessary, which is outside of the scope of the present work.

The universe age became close to the annihilation time near the hydrogen recombination era at $z=1100$ or earlier. The annihilation could distort the CMB frequency spectrum if the annihilation proceeded below $z=10^{6}$. However, the energy of the photons produced by $p \bar{p}$ or even $e^{-} e^{+}$annihilations is by far above the CMB energy at this epochs. Moreover, we believe that practically all antimatter was confined inside BD stars and very little annihilation proceeded on the star surface. However, e.g. a helium BD-star with the mass $M_{\mathrm{He}} \sim 2.5 M_{\odot}$, would evolve only about 500000 years, so most of the mass-loss would occur shortly after recombination, when the universe age was comparable to the annihilation time so the annihilation energy would interfere with the recombination dynamics, which may lead to some observable effects to be studied.

\section{E. Meteor observations}

As was mentioned above in Sec. II, (some of) the BD-stars may potentially have anomalous chemical abundances due to different values of the baryon asymmetry parameter $\beta$ during the primordial nucleosynthesis. If $\beta$ is low, no significant differences as compared to the standard BBN abundances are expected. Such 'metal-free' BD-stars should be similar to the first Population III stars. Not much dust is expected to be formed during evolution of these stars. However, in domains with large $\beta$ the initial metal abundance can be higher. These 'metal-rich' BD-stars in due course of the evolution can produce a certain fraction of solids mostly in the form of dust particles. These dust particles should move with virial halo velocities $v \sim 500 \mathrm{~km} / \mathrm{s}$ and can be observed as 'anti-meteors'. An antimeteor with mass $m$ intruding the Earth atmosphere should produce a prolific gamma-ray emission with a fluence of about $F_{\gamma} \sim 10(\mathrm{~m} / 1 \mathrm{mg}) \mathrm{erg} / \mathrm{cm}^{2}$ on a time-scale of the Earth atmosphere crossing $\sim 0.1-1 \mathrm{~s}$. The non-detection of such bright flares by all-sky gamma-ray monitors from the Earth atmosphere and from the Moon 1 led Fargion and Khlopov [56] to infer the upper bound on the antimatter fraction in meteors to be $f<10^{-8}-10^{-9}$, which is derived by assuming the complete symmetry between matter and antimatter. (Note that short, millisecond, intensive hard gamma-ray flashes with energy $10^{8}-10^{9}$ ergs are observed from the atmosphere (the so-called 'terrestrial gamma-ray flashes', TGF) [57] and are thought to be associated with atmospheric electricity [58].)

An extragalactic meteor intruding the Earth atmosphere with a velocity of at least $300 \mathrm{~km} / \mathrm{s}$ was reported in ref. [59]. The spectrum of this faint meteor was similar to the standard metal-rich chondrite. The authors [59] concluded that the space number density of such rapid dust particles, apparently of extragalactic origin, in the vicinity of the Earth can be as high as $n_{d} \sim 4 \times 10^{-26} \mathrm{~cm}^{-3}$. The (very model-dependent) estimate of the observed meteor mass is $m \sim 10^{-6} \mathrm{~g}$. The prolific metal-rich extragalactic dust particles is quite enigmatic from the point of view of standard stellar evolution. If this event were an anti-meteor, the associated gamma-ray fluence, assuming a

\footnotetext{
1 The brightest gamma-ray bursts observed by BATSE have the fluence of $\sim 10^{-3} \mathrm{erg} / \mathrm{cm}^{2}[55]$.
} 
$500 \mathrm{~km}$ distance to the space detector, would be about $10^{-2} \mathrm{erg} / \mathrm{cm}^{2}$, which is several orders of magnitude higher than the brightest BATSE flashes.

According to the standard belief the first stars ejected molecules and dust. To form larger pieces of matter (meteors) such gas should be compressed e.g. by a shock wave from supernova explosion or by a collision with another molecular cloud. As a result dust particles could be squeezed forming larger stones or pieces of ice forming protoplanetary or protostar clouds. However, the BD stars not necessarily passed through such cycles but most probably remained primary (PopIII) stars. In this case one should not expect large number of meteors from them in our neighborhood.

Thus in the context of BD-stars under scrutiny the anti-matter restrictions derived in [56] from meteor observations cannot be directly applied. Indeed, most of the cosmic anti-dust produced by mass-loss from 'metal-rich' BD-stars is expected to be in the form of small micron-size grains with a mass of $10^{-6} \mathrm{~g}$ and smaller, like the ordinary interstellar dust. Estimates made in [56] show that these small anti-dust grains should completely annihilate when moving through the interstellar gas, contributing to the diffusive gamma-ray background. Guided by the analogy with the ordinary matter, the fraction of dust in the total stellar mass should be smaller than $\sim 1 \%$ [27], thus providing only minor contribution to the observed gamma-ray background discussed above.

\section{DISCUSSION AND CONCLUSIONS}

We conclude that the contemporary observational data do not exclude significant amount of antimatter in the Galaxy (and in other galaxies), especially in the form of the baryo-dense low-massive stars created in the very early universe. The total mass of these antimatter objects could be comparable with the total mass of the Galaxy. They would populate the galactic halo and might make a noticeable contribution to dark matter and, in particular, to Machos observed through microlensing. The BD stars should have an unusual chemical content because they were formed in the regions with very high baryon-to-photon ratio, where BBN proceeded with more efficient synthesis of heavy elements. Thus a star with chemical anomaly may present a good possibility to be an antimatter star.

As we have shown above, the diffusive gamma-ray background may impose stringent constraints on the BD-star fraction because of inevitable mass-loss during evolution of the anti-helium stars. The precise amount of mass lost during evolution of an (anti) helium star depends upon its mass and metal abundance (see e.g. [28] for more detail). For example, for solar metal abundance if $M_{\mathrm{He}} \leq 0.3 M_{\odot}$, helium ignition into carbon is impossible, and the star simply cools down to form an anti-helium white dwarf without mass-loss. It is also known that for $M_{\mathrm{He}} \leq 0.8 M_{\odot}$ no significant mass loss is expected, and the star is evolved to form a hybrid CO-He white dwarf [28]. Helium stars with initial masses $0.8<M_{\mathrm{He}} / M_{\odot} \leq 2.2$ evolve to form a CO white dwarf with a mass up to $1.2 M_{\odot}$, i.e. the mass-loss from helium stars in this mass interval increases from 0 to about $45 \%$. Cores of more massive helium stars are expected to collapse to form a neutron star or black hole (for the most massive stars). Whether the collapse into a black hole is accompanied with substantial mass loss is unclear. The nuclear evolution of more massive helium stars occurs on the timescale $\tau_{\mathrm{He}} \sim 10^{7.15}\left(M_{\mathrm{He}} / M_{\odot}\right)^{-3.7}$ yrs [29]. Therefore, for interesting He-star masses $M_{\mathrm{He}} \gtrsim 0.8 M_{\odot}$, most of the possible mass loss is expected to occur in the first 60 mln years, i.e. at redshifts $z \gtrsim 43$ (for the standard cosmology), long before the formation of first structures in the universe. Assuming a homogeneous medium with the density $n(z)=n_{0}(1+z)^{3}$, where $n_{0}=\varrho_{b} / m_{p} \sim 2 \cdot 10^{-7} \mathrm{~cm}^{-3}$ is the present-day baryon number density, taking the annihilation cross section to be $\sigma_{\text {ann }} \sim 10^{-24}(\mathrm{c} / v) \mathrm{cm}^{2}$ (without account for the Sommerfeld low-velocity enhancement), and using the thermal velocity $v_{\mathrm{th}} \sim 10^{5} \sqrt{T / 10^{4} K} \mathrm{~cm} / \mathrm{s}$ in adiabatically cooling ideal mono-atomic gas with $T(z)=3 \times 10^{3}\left[(1+z) / 10^{3}\right]^{2} \mathrm{~K}$, we can estimate the annihilation time $t_{\mathrm{ann}} \sim 1 /\left(n \sigma_{\mathrm{ann}} v\right)$ to be much longer than the Hubble time at the corresponding $z$. This implies that most of the gas anti-matter from BD stellar winds is likely to survive until the present time. Even at the present time, in dense intercluster gas with baryon number density $n \sim 10^{-3} \mathrm{~cm}^{-3}$, such anti-particles moving with virial velocities of a few $1000 \mathrm{~km} / \mathrm{s}$ can annihilate only very slowly.

The physics of BD-stars is quite poorly studied and may be very much different from the usual astrophysics because the initial states of such stars quite often were different from the initial states of the usual stars. For example, BD-stars could be formed in the state when the external pressure was larger than the internal one. Moreover they start from already dense and hot state but not from cold disperse gas cloud. In particular, there could be BD-stars which are similar to the core of red giants but without external layers and some other strange objects, see discussion in ref. [15].

Let us note that allowing some fraction of the anti-matter to annihilate at high redshift may contribute to the hard radiation continuum which is necessary to the secondary ionization of the Universe at $z>10$.

As is discussed in the previous papers, BD stars may have age comparable to the universe age, and due to an anomalous initial chemistry they even might look older than the universe if their age is determined by the usual methods under assumption of the standard initial chemistry. This problem is under investigation. The observed high-redshift supernovae and gamma-ray bursts also nicely fit into the frameworks of the model.

As a by-product, the model considered here explains the formation of superheavy black holes and suggests an inverted process of the galaxy formation: first, a superheavy $\mathrm{BH}$ was born, which served as a seed for the 
subsequent collection of matter making galaxy.

\section{ACKNOWLEDGMENTS}

We are grateful to A.V.Zasov for useful indications on DM literature and to M.S. Pshirkov and L.R. Yungelson for discussions. SB and AD acknowledge the support of the grant of the Russian Federation government 11.G34.31.0047. The work of KP was supported by the Russian Science Foundation grant 14-12-00203.

[1] A. D. Sakharov, Pisma v Zhurnal Eksperimentalnoi i Teoreticheskoi Fiziki 5, 32 (1967); Soviet Journal of Experimental and Theoretical Physics Letters 5, 24 (1967).

[2] T. D. Lee, Phys.Rep. 9, 143 (1974)

[3] A. G. Cohen, A. De Rújula, and S. L. Glashow, ApJ 495, 539 (1998), astro-ph/9707087

[4] M. Sasaki, S. Haino, K. Abe, and others, Advances in Space Research 42, 450 (2008).

[5] M. Boezio and PAMELA Collaboration, Journal of Physics Conference Series 110, 062002 (2008).

[6] J. Alcaraz, D. Alvisi, B. Alpat, and others, Physics Letters B 461, 387 (1999), hep-ex/0002048

[7] R. Duperray, B. Baret, D. Maurin, and others, Phys. Rev. D. 71, 083013 (2005), astro-ph/0503544

[8] N. Martin and ALICE Collaboration, Journal of Physics Conference Series 455, 012007 (2013)

[9] A. Kalweit, Light hyper- and anti-nuclei production at the LHC measured with ALICE (2014), https://indico.cern.ch/event/328442.

[10] G. Steigman, ARA\&A 14, 339 (1976).

[11] G. Steigman, J. Cosmol. Astropart. Phys. 10, 001 (2008), arXiv:0808.1122,

[12] P. von Ballmoos, Hyperfine Interactions (2014), 10.1007/s10751-014-1024-9, arXiv:1401.7258 [astro-ph.HE].

[13] A. Dolgov and J. Silk, Phys. Rev. D. 47, 4244 (1993)

[14] A. D. Dolgov, M. Kawasaki, and N. Kevlishvili, Nuclear Physics B 807, 229 (2009), arXiv:0806.2986.

[15] C. Bambi and A. D. Dolgov, Nuclear Physics B 784, 132 (2007) arXiv:astro-ph/0702350.

[16] A. D. Dolgov, I. B. Khriplovich, and A. S. Rudenko, Soviet Journal of Experimental and Theoretical Physics Letters 96, 421 (2012) arXiv:1208.3565 [astro-ph.CO]

[17] A. D. Dolgov, V. A. Novikov, and M. I. Vysotsky, Soviet Journal of Experimental and Theoretical Physics Letters 98, 519 (2014) arXiv:1309.2746 [hep-ph]

[18] A. D. Dolgov and S. I. Blinnikov, Phys. Rev. D. 89, 021301 (2014), arXiv:1309.3395 [astro-ph.CO]

[19] M. Y. Khlopov, S. G. Rubin, and A. S. Sakharov, Phys. Rev. D. 62, 083505 (2000) hep-ph/0003285.

[20] M. Y. Khlopov, S. G. Rubin, and A. S. Sakharov, ArXiv High Energy Physics - Phenomenology e-prints (2002), hep-ph/0210012.

[21] L. Canetti, M. Drewes, and M. Shaposhnikov, New Journal of Physics 14, 095012 (2012), arXiv:1204.4186 [hep-ph].

[22] I. Affleck and M. Dine, Nuclear Physics B 249, 361 (1985).

[23] S. Coleman and E. Weinberg, Phys. Rev. D. 7, 1888 (1973)

[24] A. Seth, R. van den Bosch, S. Mieske, and others, Nature 513, 398 (2014), arXiv:1409.4769.

[25] R. Nakamura, M.-a. Hashimoto, S.-i. Fujimoto, N. Nishimura, and K. Sato, ArXiv e-prints (2010), arXiv:1007.0466

[26] P. Sreekumar, D. L. Bertsch, B. L. Dingus, and others, ApJ 494, 523 (1998) astro-ph/9709257, A. W. Strong, I. V. Moskalenko, and O. Reimer, ApJ 613, 956 (2004), astro-ph/0405441.

[27] M. Fukugita, C. J. Hogan, and P. J. E. Peebles, ApJ 503, 518 (1998), astro-ph/9712020. M. Fukugita and P. J. E. Peebles, ApJ 616, 643 (2004), astro-ph/0406095.

[28] I. Iben, Jr. and A. V. Tutukov, ApJS 58, 661 (1985).

[29] G. Nelemans, S. F. Portegies Zwart, F. Verbunt, and L. R. Yungelson, A\&A 368, 939 (2001), astro-ph/0101123

[30] J. A. R. Caldwell and J. P. Ostriker, ApJ 251, 61 (1981)

[31] K. Griest, Phys. Rev. D. 37, 2703 (1988)

[32] A. M. Green, Modern Physics Letters A 27, 1230004 (2012), arXiv:1112.0524 [astro-ph.CO]

[33] P. J. T. Leonard and S. Tremaine, ApJ 353, 486 (1990).

[34] M. C. Smith, G. R. Ruchti, A. Helmi, and others, MNRAS 379, 755 (2007), astro-ph/0611671

[35] V. S. Berezinskii, S. V. Bulanov, V. A. Dogiel, and V. S. Ptuskin, Amsterdam: North-Holland, 1990, edited by Ginzburg, V.L. (1990).

[36] R. Blandford and D. Eichler, Phys.Rep. 154, 1 (1987)

[37] F. C. Jones and D. C. Ellison, Space Sci. Rev. 58, 259 (1991).

[38] A. R. Bell, MNRAS 353, 550 (2004)

[39] A. M. Bykov and I. N. Toptygin, Astronomy Letters 31, 748 (2005), A. Vladimirov, D. C. Ellison, and A. Bykov, ApJ 652, 1246 (2006), astro-ph/0606433 A. M. Bykov and I. Toptygin, Physics Uspekhi 50, 141 (2007) A. M. Bykov, D. C. Ellison, and M. Renaud, Space Sci. Rev. 166, 71 (2012), arXiv:1105.0130 [astro-ph.HE]

[40] Z. G. Berezhiani, A. D. Dolgov, and R. N. Mohapatra, Phys. Lett. B 375, 26 (1996) arXiv:hep-ph/9511221

[41] S. I. Blinnikov, (1998), arXiv:astro-ph/9801015

[42] C. Alcock, R. A. Allsman, D. R. Alves, and others, Astrophys. J. 542, 281 (2000), arXiv:astro-ph/0001272

[43] D. P. Bennett, Astrophys. J. 633, 906 (2005), arXiv:astro-ph/0502354 
[44] P. Tisserand, L. Le Guillou, C. Afonso, and others, A\&A 469, 387 (2007), astro-ph/0607207

[45] A. Riffeser, S. Seitz, and R. Bender, Astrophys. J. 684, 1093 (2008), arXiv:0805.0137 [astro-ph].

[46] G. Ingrosso, S. Calchi Novati, F. de Paolis, and others, A\&A 462, 895 (2007), astro-ph/0610239

[47] M. Moniez, General Relativity and Gravitation 42, 2047 (2010), arXiv:1001.2707 [astro-ph.GA]

[48] S. Calchi Novati, S. Mirzoyan, P. Jetzer, and G. Scarpetta, MNRAS 435, 1582 (2013), arXiv:1308.4281 [astro-ph.GA].

[49] S. Mao, Research in Astronomy and Astrophysics 12, 947 (2012) arXiv:1207.3720 [astro-ph.GA]

[50] J. Yoo, J. Chanamé, and A. Gould, Astrophys. J. 601, 311 (2004), astro-ph/0307437.

[51] N. W. Evans and V. Belokurov, MNRAS 374, 365 (2007).

[52] A. V. Byalko, Astron. Zh. 46, 998 (1969); Sov. Astron. 13, 784 (1970).

[53] B. Paczynski, ApJ 304, 1 (1986).

[54] D. P. Quinn, M. I. Wilkinson, M. J. Irwin, and others, arXiv: (2009), 0903.1644 [astro-ph]

[55] W. S. Paciesas, C. A. Meegan, G. N. Pendleton, and others, ApJS 122, 465 (1999) astro-ph/9903205

[56] D. Fargion and M. Khlopov, Astroparticle Physics 19, 441 (2003), hep-ph/0109133.

[57] G. J. Fishman, P. N. Bhat, R. Mallozzi, and others, Science 264, 1313 (1994)

[58] J. R. Dwyer, D. M. Smith, and S. A. Cummer, Space Sci. Rev. 173, 133 (2012)

[59] V. L. Afanasiev, V. V. Kalenichenko, and I. D. Karachentsev, Astrophysical Bulletin 62, 301 (2007), arXiv:0712.1571. 